EP-18

\title{
Surgical anatomy of caudate bile ducts: An important technique to enhance enhance cholangiogram interpretation
}

Rajeev SINHA*

Department of Hepatic and Pancreatic Surgery, Medical College, India

Introduction: Caudate bile ducts are routinely presented using negative images as X-ray-cholangiograms. Such information does not provide for instant surgical orientation of the relationships between caudate ducts and the liver itself-a paramount skill for successfully performing hilar cholangiocarcinoma resection and living donor/split transplantation. This study presents a 4-step procedure to prepare, dissect and present, high-quality, two-dimensional/three-dimensional (2D/3D) anatomical images of biliary caudate ducts in a surgically meaningful way.

Methods: Fresh cadavers had arteries and veins injected with colored-silicone and ducts bile-stained to facilitate recognition. Dissections were performed under magnification with microsurgical instruments. Stepwise 2D and 3D images were acquired

Results: Dissection of silicone-injected specimens under magnification allows identification of caudate structures, its portions and processes while preserving tridimensional arrangement of caudate vessels, biliary ducts and collectors. Such dissections can help enhance cholangiogram interpretation and favor its direct correlation to intraoperative findings

Conclusions: A procedure including: a) preparation of high-quality cadaveric livers; b) with silicone-injected vessels; c) dissected under surgical microscope; and d) documented using 2D and 3D images aimed at enhancing the clinical understanding of the anatomy of caudate ducts is presented. It has potential to enhance morphological and clinical understanding of caudate ducts, being useful to anatomists and surgeons alike. 